\title{
In silico identification of GPI-anchored proteins in Paracoccidioides
}

Basso, L.R. Jr.*, Gonçales, R.A.*, Vasconcelos, E.J.R, Reis, T.F., Ruy, P. C., Ruiz, J.C., and Coelho P.S.R. ${ }^{1,2}$

1- Department of Cellular and Molecular Biology and Pathogenic Bioagents, Faculty of Medicine of Ribeirão Preto - University of São Paulo - USP - Brazil

2. Corresponding author.

* These authors contributed equally to this work

Mailing address: Department of Cellular and Molecular Biology and Pathogenic Bioagents, Faculty of Medicine of Ribeirão Preto - University of São Paulo - USP, Avenida dos Bandeirantes, 3900, Ribeirão Preto, SP 14049-900

Fax (55) 16 3315-3257. E-mail: pacoelho@ fmrp.usp.br 


\section{ABSTRACT}

Glycosylphosphatidylinositol-anchored proteins (GPI-proteins) are widely found in eukaryotic organisms. In fungi, GPI-proteins are thought to be involved in diverse cellular mechanisms such as cell wall biosynthesis and cell wall remodeling, adhesion, antigenicity, and virulence. The conserved structural domains of GPI-protein allow the utilization of in silico prediction approach to identify this class of proteins using a genome-wide analysis. We used different previously characterized algorithms to search for genes that encode predicted GPIproteins in the genome of $P$. brasiliensis and $P$. lutzii, thermal dimorphic fungi that causes paracoccidioidomycosis (PCM). By using these methods, 98 GPI-proteins were found in $P$. brasiliensis with orthologs in P. lutzii. A series of 28 GPI-proteins were classified in functional categories (such as glycoside hydrolases, chitin-processing proteins, and proteins involved in the biogenesis of the cell wall). Furthermore, 70 GPI-proteins exhibited homology with hypothetical conserved proteins of unknown function. These data will be an important resource for the future analysis of GPI-proteins in Paracoccidioides spp.

Keywords: Paracoccidioidomycosis, Paracoccidioides brasiliensis, Paracoccidioides lutzii, Cell wall, Glycosylphosphatidylinositol-anchored proteins 


\section{Introduction}

In pathogenic fungi, cell wall components mediate the contact, adhesion, and invasion of the host tissue (Levitz, 2010) which is characterized by a distinctive composition depending on the morphological phase. The covalently linked glycosylphosphatidylinositol (GPI)-proteins represents the main class of proteins in the cell wall. Members of this group are pivotal players in cell wall biogenesis and remodeling and in essential pathogenic processes such as adhesion to and degradation of host tissues (Hube and Naglik, 2001; Martchenko et al., 2004; Richard et al., 2002; Richard and Plaine, 2007; Sundstrom, 2002). All GPI-proteins show common characteristics such as a $\mathrm{C}$-terminal consensus sequence for GPI modification and an N-terminal signal peptide for translocation across the membrane of the endoplasmic reticulum. In addition, many GPI-proteins are heavily glycosylated. These well-defined characteristics allow the use of in silico approaches for screening and identification of GPI-proteins in the genome (De Groot et al., 2003; Eisenhaber et al., 2004).

The Paracoccidioides genus includes species of thermodimorphic pathogenic fungi that cause paracoccidioidomycosis (PCM) which is the most prevalent systemic human mycosis occurring in Latin America. Multilocus and genomic sequencing studies indicate the existence of two distinct species within the Paracoccidioides genus, $P$. brasiliensis and P. lutzii (4). $P$. brasiliensis is comprised of distinct lineages (S1, PS2, PS3, and PS4) that cause most of PCM cases throughout South America (4-6). Paracoccidioides lutzii isolates are found predominantly in north, central and southwest of Brazil and Ecuador (Teixeira et al., 2009; Theodoro et al., 2012).

The complete genome sequence of three strains ( $\mathrm{Pb} 18, \mathrm{~Pb} 03$ and $\mathrm{Pb} 01$-like) from the Paracoccidioides genus (Desjardins et al., 2011a; Munoz et al., 2014) have been recently available. The genomic information has allowed the identification, in $P$. brasiliensis and $P$. lutzii, of a group of 62 predicted GPI-proteins based on an in silico analysis using one program of prediction (Desjardins et al., 2011a, b). Based in the current genome sequence of $P$. brasiliensis and P. lutzii we used five algorithms to look for additional GPI-proteins that may not have been detected by the previous genome analysis. The identification of new $P$. brasiliensis GPI-proteins may reveal targets to be used in the future for the development of new diagnostic tools, drugs, and immunologic tests. 


\section{Material and Methods}

\section{In silico identification of predicted GPI-proteins and transcription analysis}

The pipeline for the identification of GPI-protein coding genes consisted of the following steps: (1) A proteome file containing 8741 ORF sequences of $P$. brasiliensis strain Pb18 and 9.132 ORF sequences of $P$. lutzii (Desjardins et al., 2011a, b) were downloaded from the Broad Institute website (http://www.broad.mit.edu) last accessed on March 2013. These proteomes were searched for proteins carrying a GPI anchor signal peptide (omega site) using five different methods: one developed by de Groot and collaborators (De Groot et al., 2003), Big-PI ( http:// Eisenhaber et al., 2004), GPI-SOM ( http:// Fankhauser and Maser, 2005), PredGPI ( http://Pierleoni et al., 2008a, b) and FragAnchor ( http:// Poisson et al., 2007). predicted-GPI proteins detected by at least two methods were selected for further analysis. Protein sequences from $P$. brasiliensis and $P$. lutzii that presented mismatches after alignment by ClustalW were reannotated (see the following section) (3) Proteins were searched for the presence of an N-terminal ER-import signal by SignalP V3.0 software available at (http://www.cbs.dtu.dk/services/SignalP-3.0/). The standardized threshold value of 0.5 was applied for signal peptides in the two algorithms [SignalP-NN (Smean) and SignalP-HMM (Sprob)]. (4) The presence of internal membrane domains and protein localization was investigated using TMHMM (http://www.cbs.dtu.dk/services/TMHMM/) and PSORTII (http://psort.nibb.ac.jp).

Predicted GPI proteins were used as queries in BLASTp searches (Altschul et al., 1990) to identify sequence similarities in 17 other fungal genomes (Cuomo and Birren, 2010). After the alignment with ClustalW (http://www.genome.jp/tools/clustalw), we considered orthologues when their protein sequence presents $>30 \%$ of amino acid identity in a region encompassing $>60 \%$ of the whole sequence. BLASTP searches were performed at NCBI (http://www.ncbi.nlm.nih.gov/blast).

For transcription analysis, ESTs were manually searched for hits in the Broad Institute database.

\section{Annotation and manual curation of GPI-protein coding genes}

Genomic DNA sequences encompassing ORFs coding for GPI-anchoring signals from $1000 \mathrm{bp}$ upstream the start codon to $1000 \mathrm{bp}$ downstream the stop codon - were analysed by five programs to determine the exon-intron map: Augustus (http://bioinf.unigreifswald.de/augustus/), Fgenesh (http://linux1.softberry.com), GeneMark 
(http://opa.biology.gatech.edu/GeneMark/), Geneid (http://genome.crg.es/geneid.html) and Genscan (http://genes.mit.edu/GENSCAN.html),. The consensus gene sequence from all prediction strategies was aligned by ClustalW with the orthologues of P. brasiliensis ( $\mathrm{Pb} 18$, $\mathrm{Pb} 03)$ and $P$. lutzii $(\mathrm{Pb} 01)$ whose sequences are from the NCBI fungal data bank. The annotated sequences were re-analyzed to confirm the presence of GPI-anchoring signal and signal peptide, and absence of transmembrane domains as described in the previous section.

\section{Results and Discussion}

\section{In silico identification of GPI proteins in $P$. brasiliensis}

Five different in silico methods were used to comprehensively identify putative GPI proteins in the P. brasiliensis and P. lutizi genome (De Groot et al., 2003; Eisenhaber et al., 2004; Fankhauser and Maser, 2005; Pierleoni et al., 2008a; Poisson et al., 2007). The resulting genomic sequences were reannotaded when there was a discrepancy in the exon-map structure after alignment with conserved genomic sequences from close relatives (e.g $P$. brasiliensis $\mathrm{X}$ P. lutzii; see materials and methods and supplementary data). We identified 28 GPI-proteins that can be classified into 5 functional categories, 70 conserved proteins with unknown functions, in a total of 98 GPI-proteins (Table 1). The ratio of predicted GPI-proteins (1.12\%) to the proteome is similar to that observed in filamentous fungi and yeasts (de Groot et al., 2009; de Groot et al., 2003; Desjardins et al., 2011; Eisenhaber et al., 2004). We did not find differences in the number of GPI-proteins homologs between P. brasiliensis and P. lutzii.

\section{Synthesis and processing of glycan, chitin and other enzymatic processes of the cell wall}

Sixteen of the predicted GPI-proteins have homology with glycosyl hydrolases (GH), (Cantarel et al., 2009; Lairson et al., 2008). Three proteins belong to the GH72 family or Gel/Gas/Phr of 1,3- $\beta$ - glucanosyltransferases (Castro Nda et al., 2009; Mouyna et al., 2000; Popolo et al., 2008). Four GPI-proteins belong to the Crh (GH16) family which consists mainly of 1,3- $\beta$-glucanases and 1,3-1,4- $\beta$-glucanosyltransferases (Cabib et al., 2008). Three members of the Dfg5 (GH76) family were also identified, and they are endomananases with a putative role in the incorporation of GPI-proteins to the cellular wall (Maddi et al., 2012). An alfaamylase (GH13) is present and has a putative role of in the processing of 1,3- $\alpha$-glucana/1,4- $\alpha$ glucana (Camacho et al., 2012). The PbPga1 is a cell wall protein with immunogenic properties and is recognized specifically by serum of patients with paracoccidioidomycosis (Valim et al., 2012). 

enzyme in fungi has not yet been determined. It can be speculated that the molecule can serve as a tool in the breakage of the bacterial peptidoglycan for nutritional purposes (Korczynska et al., 2010). An N-acetyl- $\beta$-D-glicosaminidase (GH20) was identified (Santos et al., 2004) and is a member of the GH92 family. Finally, it was identified a member of the GH3 family, a glucosyl-transferase (GT1) and a cutinase (CE5). Cutinases are extracellular enzymes secreted by microorganisms to break the cellular wall of plants (Lin and Kolattukudy, 1980). The protein is possibly a false positive because these enzymes are secreted (de Vries, 2003).

\section{Cell wall biogenesis}

One of the proteins identified (Ecm33p) is thought to play an important role in cell wall biogenesis. This protein shares homology with the Ecm33p family, also a ubiquitous fungal GPI protein family. Deletion of ECM33 in S. cerevisiae results in swollen cells that secrete increased levels of 1,6- $\beta$-glucosylated CWPs into the culture medium and are hypersensitive to drugs that interfere with cell wall biosynthesis (De Groot et al., 2001).

\section{Proteins with varied functions}

Four proteins share homology with fungal proteins not directly associated with the cell wall. We identified two superoxide dismutases (SOD). SOD converts superoxide radicals into hydrogen peroxide and molecular oxygen and is thought to be important to the pathogenesis of fungal infection. Two proteins share homology with hydrophobins, which in filamentous fungi play a role in the formation of aerial hyphae and in the attachment of hyphae to hydrophobic surfaces. The protein PbPga1 was first identified by homology with a predicted GPI-protein of Histoplasma capsulatum. PbPgal has been characterized as a cell surface protein recognized

\section{Conservation of Paracoccidioides brasiliensis GPI- proteins}

We analyzed the conservation of predicted GPI proteins of known function in 17 different fungal genomes. These proteins represent the most conserved proteins (Fig 1) and are involved in different biosynthetic and processing pathways (glycosylases, proteases and lipases). On the other hand, the less conserved proteins (Fig 2) are those with unannotated function. These proteins may not have catalytic roles in the cell walls. Many of the identified proteins with unknown function are expressed as evidenced by EST hits in databases (Table 1) 


\section{References}

Albuquerque, C.F., da Silva, S.H., and Camargo, Z.P. (2005). Improvement of the specificity of an enzyme-linked immunosorbent assay for diagnosis of paracoccidioidomycosis. $\mathrm{J}$ Clin Microbiol 43, 1944-1946.

Albuquerque, P., Kyaw, C.M., Saldanha, R.R., Brigido, M.M., Felipe, M.S., and SilvaPereira, I. (2004). Pbhyd1 and Pbhyd2: two mycelium-specific hydrophobin genes from the dimorphic fungus Paracoccidioides brasiliensis. Fungal Genet Biol 41, 510-520.

Altschul, S.F., Gish, W., Miller, W., Myers, E.W., and Lipman, D.J. (1990). Basic local alignment search tool. J Mol Biol 215, 403-410.

Braun, B.R., Head, W.S., Wang, M.X., and Johnson, A.D. (2000). Identification and characterization of TUP1-regulated genes in Candida albicans. Genetics 156, 31-44.

Cabib, E., Blanco, N., Grau, C., Rodriguez-Pena, J.M., and Arroyo, J. (2007). Crh1p and $\mathrm{Crh} 2 \mathrm{p}$ are required for the cross-linking of chitin to beta(1-6)glucan in the Saccharomyces cerevisiae cell wall. Mol Microbiol 63, 921-935.

Cabib, E., Farkas, V., Kosik, O., Blanco, N., Arroyo, J., and McPhie, P. (2008). Assembly of the yeast cell wall. Crh1p and Crh2p act as transglycosylases in vivo and in vitro. J Biol Chem 283, 29859-29872.

Camacho, E., Sepulveda, V.E., Goldman, W.E., San-Blas, G., and Nino-Vega, G.A. (2012). Expression of Paracoccidioides brasiliensis AMY1 in a Histoplasma capsulatum amy1 mutant, relates an alpha-(1,4)-amylase to cell wall alpha-(1,3)-glucan synthesis. PLoS One 7, e50201.

Castro Nda, S., de Castro, K.P., Orlandi, I., Feitosa Ldos, S., Rosa e Silva, L.K., Vainstein, M.H., Bao, S.N., Vai, M., and Soares, C.M. (2009). Characterization and functional analysis of the beta-1,3-glucanosyltransferase 3 of the human pathogenic fungus Paracoccidioides brasiliensis. FEMS Yeast Res 9, 103-114.

Cuomo, C.A., and Birren, B.W. (2010). The fungal genome initiative and lessons learned from genome sequencing. Methods Enzymol 470, 833-855.

De Groot, P.W., Hellingwerf, K.J., and Klis, F.M. (2003). Genome-wide identification of fungal GPI proteins. Yeast 20, 781-796.

Desjardins, C.A., Champion, M.D., Holder, J.W., Muszewska, A., Goldberg, J., Bailao, A.M., Brigido, M.M., Ferreira, M.E., Garcia, A.M., Grynberg, M., et al. (2011a). Comparative genomic analysis of human fungal pathogens causing paracoccidioidomycosis. PLoS Genet 7, 27.

Desjardins, C.A., Champion, M.D., Holder, J.W., Muszewska, A., Goldberg, J., Bailao, A.M., Brigido, M.M., Ferreira, M.E., Garcia, A.M., Grynberg, M., et al. (2011b). Comparative genomic analysis of human fungal pathogens causing paracoccidioidomycosis. PLoS Genet 7, e1002345.

Dugger, K.O., Villareal, K.M., Ngyuen, A., Zimmermann, C.R., Law, J.H., and Galgiani, J.N. (1996). Cloning and sequence analysis of the cDNA for a protein from Coccidioides immitis with immunogenic potential. Biochem Biophys Res Commun 218, 485-489.

Eisenhaber, B., Schneider, G., Wildpaner, M., and Eisenhaber, F. (2004). A sensitive predictor for potential GPI lipid modification sites in fungal protein sequences and its application to genome-wide studies for Aspergillus nidulans, Candida albicans, Neurospora crassa, Saccharomyces cerevisiae and Schizosaccharomyces pombe. J Mol Biol 337, 243-253.

Fankhauser, N., and Maser, P. (2005). Identification of GPI anchor attachment signals by a Kohonen self-organizing map. Bioinformatics 21, 1846-1852. 
Ghannoum, M.A., Spellberg, B., Saporito-Irwin, S.M., and Fonzi, W.A. (1995). Reduced virulence of Candida albicans PHR1 mutants. Infect Immun 63, 4528-4530.

Gomez, B.L., Figueroa, J.I., Hamilton, A.J., Ortiz, B., Robledo, M.A., Hay, R.J., and Restrepo, A. (1997). Use of monoclonal antibodies in diagnosis of paracoccidioidomycosis: new strategies for detection of circulating antigens. J Clin Microbiol 35, 3278-3283.

Hube, B., and Naglik, J. (2001). Candida albicans proteinases: resolving the mystery of a gene family. Microbiology 147, 1997-2005.

James, T.Y., Kauff, F., Schoch, C.L., Matheny, P.B., Hofstetter, V., Cox, C.J., Celio, G., Gueidan, C., Fraker, E., Miadlikowska, J., et al. (2006). Reconstructing the early evolution of Fungi using a six-gene phylogeny. Nature 443, 818-822.

Kanetsuna, F., Carbonell, L.M., Moreno, R.E., and Rodriguez, J. (1969). Cell wall composition of the yeast and mycelial forms of Paracoccidioides brasiliensis. $\mathbf{J}$ Bacteriol 97, 1036-1041.

Kubista, M., Andrade, J.M., Bengtsson, M., Forootan, A., Jonak, J., Lind, K., Sindelka, R., Sjoback, R., Sjogreen, B., Strombom, L., et al. (2006). The real-time polymerase chain reaction. Mol Aspects Med 27, 95-125.

Kulkarni, R.D., Kelkar, H.S., and Dean, R.A. (2003). An eight-cysteine-containing CFEM domain unique to a group of fungal membrane proteins. Trends Biochem Sci 28, 118 121.

Lacaz, C.D., Minami, P.S., and Ramos, W.F. (1963). [Morphological Aspects of Paracoccidioides Brasiliensis in Parasitic Life]. Rev Hosp Clin Fac Med Sao Paulo 18, 273-284.

Levitz, S.M. (2010). Innate recognition of fungal cell walls. PLoS Pathog 6, 1000758.

Lin, T.S., and Kolattukudy, P.E. (1980). Structural studies on cutinase, a glycoprotein containing novel amino acids and glucuronic acid amide at the $\mathrm{N}$ terminus. Eur $\mathrm{J}$ Biochem 106, 341-351.

Linder, M.B., Szilvay, G.R., Nakari-Setala, T., and Penttila, M.E. (2005). Hydrophobins: the protein-amphiphiles of filamentous fungi. FEMS Microbiol Rev 29, 877-896.

Martchenko, M., Alarco, A.M., Harcus, D., and Whiteway, M. (2004). Superoxide dismutases in Candida albicans: transcriptional regulation and functional characterization of the hyphal-induced SOD5 gene. Mol Biol Cell 15, 456-467.

Martinez-Lopez, R., Monteoliva, L., Diez-Orejas, R., Nombela, C., and Gil, C. (2004). The GPI-anchored protein $\mathrm{CaEcm} 33 \mathrm{p}$ is required for cell wall integrity, morphogenesis and virulence in Candida albicans. Microbiology 150, 3341-3354.

Martinez-Lopez, R., Nombela, C., Diez-Orejas, R., Monteoliva, L., and Gil, C. (2008). Immunoproteomic analysis of the protective response obtained from vaccination with Candida albicans ecm33 cell wall mutant in mice. Proteomics 8, 2651-2664.

Martinez-Lopez, R., Park, H., Myers, C.L., Gil, C., and Filler, S.G. (2006). Candida albicans Ecm33p is important for normal cell wall architecture and interactions with host cells. Eukaryot Cell 5, 140-147.

Mendes-Giannini, M.J., Moraes, R.A., and Ricci, T.A. Proteolytic activity of the 43,000 molecular weight antigen secreted by Paracoccidioides brasiliensis (Rev Inst Med Trop Sao Paulo. 1990 Sep-Oct;32(5):384-5.).

Mouyna, I., Fontaine, T., Vai, M., Monod, M., Fonzi, W.A., Diaquin, M., Popolo, L., Hartland, R.P., and Latge, J.P. (2000). Glycosylphosphatidylinositol-anchored glucanosyltransferases play an active role in the biosynthesis of the fungal cell wall. $\mathrm{J}$ Biol Chem 275, 14882-14889.

Mouyna, I., Morelle, W., Vai, M., Monod, M., Lechenne, B., Fontaine, T., Beauvais, A., Sarfati, J., Prevost, M.C., Henry, C., et al. (2005). Deletion of GEL2 encoding for a 
beta(1-3)glucanosyltransferase affects morphogenesis and virulence in Aspergillus fumigatus. Mol Microbiol 56, 1675-1688.

Munoz, J.F., Gallo, J.E., Misas, E., Priest, M., Imamovic, A., Young, S., Zeng, Q., Clay, O.K., McEwen, J.G., and Cuomo, C.A. (2014). Genome update of the dimorphic human pathogenic fungi causing paracoccidioidomycosis. PLoS Negl Trop Dis 8.

Pardo, M., Monteoliva, L., Vazquez, P., Martinez, R., Molero, G., Nombela, C., and Gil, C. (2004). PST1 and ECM33 encode two yeast cell surface GPI proteins important for cell wall integrity. Microbiology 150, 4157-4170.

Pierleoni, A., Martelli, P.L., and Casadio, R. (2008a). PredGPI: a GPI-anchor predictor. BMC Bioinformatics 9, 1471-2105.

Pierleoni, A., Martelli, P.L., and Casadio, R. (2008b). PredGPI: a GPI-anchor predictor. BMC Bioinformatics 9, 392.

Poisson, G., Chauve, C., Chen, X., and Bergeron, A. (2007). FragAnchor: a large-scale predictor of glycosylphosphatidylinositol anchors in eukaryote protein sequences by qualitative scoring. Genomics Proteomics Bioinformatics 5, 121-130.

Popolo, L., Ragni, E., Carotti, C., Palomares, O., Aardema, R., Back, J.W., Dekker, H.L., de Koning, L.J., de Jong, L., and de Koster, C.G. (2008). Disulfide bond structure and domain organization of yeast beta(1,3)-glucanosyltransferases involved in cell wall biogenesis. J Biol Chem 283, 18553-18565.

Popolo, L., and Vai, M. (1999). The Gas1 glycoprotein, a putative wall polymer cross-linker. Biochim Biophys Acta 1426, 385-400.

Puccia, R., and Travassos, L.R. (1991). The 43-kDa glycoprotein from the human pathogen Paracoccidioides brasiliensis and its deglycosylated form: excretion and susceptibility to proteolysis. Arch Biochem Biophys 289, 298-302.

Richard, M., De Groot, P., Courtin, O., Poulain, D., Klis, F., and Gaillardin, C. (2002). GPI7 affects cell-wall protein anchorage in Saccharomyces cerevisiae and Candida albicans. Microbiology 148, 2125-2133.

Richard, M.L., and Plaine, A. (2007). Comprehensive analysis of glycosylphosphatidylinositol-anchored proteins in Candida albicans. Eukaryot Cell 6, 119-133.

Romano, J., Nimrod, G., Ben-Tal, N., Shadkchan, Y., Baruch, K., Sharon, H., and Osherov, N. (2006). Disruption of the Aspergillus fumigatus ECM33 homologue results in rapid conidial germination, antifungal resistance and hypervirulence. Microbiology 152, 1919-1928.

Santos, M.O., Pereira, M., Felipe, M.S., Jesuino, R.S., Ulhoa, C.J., Soares Rde, B., and Soares, C.M. (2004). Molecular cloning and characterization of a cDNA encoding the $\mathrm{N}$-acetyl-beta-D-glucosaminidase homologue of Paracoccidioides brasiliensis. Med Mycol 42, 247-253.

Silveira-Gomes, F., Sarmento, D.N., Pinto, T.M., Pimentel, R.F., Nepomuceno, L.B., Espirito Santo, E.P., Mesquita-da-Costa, M., Camargo, Z.P., and Marques-da-Silva, S.H. (2011). Development and evaluation of a latex agglutination test for the serodiagnosis of paracoccidioidomycosis. Clin Vaccine Immunol 18, 604-608.

Sundstrom, P. (2002). Adhesion in Candida spp. Cell Microbiol 4, 461-469.

Taborda, C.P., and Camargo, Z.P. (1994). Diagnosis of paracoccidioidomycosis by dot immunobinding assay for antibody detection using the purified and specific antigen gp43. J Clin Microbiol 32, 554-556.

Tamayo, D., Munoz, J.F., Lopez, A., Uran, M., Herrera, J., Borges, C.L., Restrepo, A., Soares, C.M., Taborda, C.P., Almeida, A.J., et al. (2016). Identification and Analysis of the Role of Superoxide Dismutases Isoforms in the Pathogenesis of Paracoccidioides spp. PLoS Negl Trop Dis 10. 
Teixeira, M.M., Theodoro, R.C., de Carvalho, M.J., Fernandes, L., Paes, H.C., Hahn, R.C., Mendoza, L., Bagagli, E., San-Blas, G., and Felipe, M.S. (2009). Phylogenetic analysis reveals a high level of speciation in the Paracoccidioides genus. Mol Phylogenet Evol 52, 273-283.

Theodoro, R.C., Teixeira Mde, M., Felipe, M.S., Paduan Kdos, S., Ribolla, P.M., San-Blas, G., and Bagagli, E. (2012). Genus paracoccidioides: Species recognition and biogeographic aspects. PLoS One 7, 30.

Valim, C.X., Basso, L.R., Jr., dos Reis Almeida, F.B., Reis, T.F., Damasio, A.R., Arruda, L.K., Martinez, R., Roque-Barreira, M.C., Oliver, C., Jamur, M.C., et al. (2012). Characterization of PbPga1, an antigenic GPI-protein in the pathogenic fungus Paracoccidioides brasiliensis. PLoS One 7, 14.

Zhu, H., Bilgin, M., Bangham, R., Hall, D., Casamayor, A., Bertone, P., Lan, N., Jansen, R., Bidlingmaier, S., Houfek, T., et al. (2001). Global analysis of protein activities using proteome chips. Science 293, 2101-2105. 
Table 1 - Predicted GPI proteins of Paracoccidioides

\begin{tabular}{|c|c|c|c|c|c|c|c|c|c|c|c|}
\hline $\begin{array}{l}\text { P. brasiliensis } \\
\text { ORF ID }\end{array}$ & $\begin{array}{l}P . \text { Iutzii } \\
\text { ORF ID }\end{array}$ & $\begin{array}{l}\text { Size (aa - } \\
\mathrm{Pb} / \mathrm{PI})\end{array}$ & $\begin{array}{c}\text { Identity } \\
(\%)\end{array}$ & $\begin{array}{l}\text { Big-PI } \\
\text { (Pb/PI) }\end{array}$ & $\begin{array}{c}\text { FragAnchor } \\
(\mathrm{Pb} / \mathrm{PI})\end{array}$ & $\begin{array}{l}\text { PredGPI } \\
\text { (Pb/PI) }\end{array}$ & $\begin{array}{l}\text { DeGroot } \\
\text { (Pb/PI) }\end{array}$ & $\begin{array}{l}\text { GPI-SOM } \\
\text { (Pb/PI) }\end{array}$ & $\begin{array}{c}\text { SignalP } \\
(\mathrm{Pb} / \mathrm{PI})\end{array}$ & EST & function/family \\
\hline \multicolumn{12}{|c|}{ Carbohydrate modifying enzymes and resident proteins } \\
\hline 04499 & 07670 & $370 / 389$ & 95.7 & + & $\mathrm{H}$ & $\mathrm{H}$ & + & + & $-/+$ & $45 / 47$ & ECM33 \\
\hline 02460 & 04708 & $225 / 225$ & 79.2 & $+/+$ & $\mathrm{H} / \mathrm{H}$ & $\mathrm{H} / \mathrm{H}$ & $+/+$ & $+/+$ & + & $9 / 7$ & PbPga1 \\
\hline 04918 & 05068 & $529 / 529$ & 97.1 & + & $\mathrm{H}$ & $\mathrm{H}$ & + & + & + & 29 & $\mathrm{GH} 2$ \\
\hline 05076 & 05245 & $471 / 471$ & 98.1 & + & $\mathrm{H}$ & $\mathrm{H}$ & + & + & + & 6 & GH72 (Gel) \\
\hline 04328 & 06867 & $224 / 224$ & 94.6 & + & $\mathrm{L} / \mathrm{H}$ & W/P & + & + & - & - & GH16 (Crh) \\
\hline 04416 & 06779 & $436 / 435$ & 77.7 & $+/-$ & $\mathrm{L} /-$ & $\mathrm{H} /-$ & $+/-$ & $+/-$ & + & 5 & GH16 (Crh) \\
\hline 06728 & 05380 & $754 / 743$ & 96.9 & $+/-$ & $\mathrm{L} /-$ & $\mathrm{H} /-$ & + & + & + & - & $\mathrm{GH} 20$ \\
\hline 03691 & 01139 & $432 / 431$ & 92.1 & $+/+$ & - & $\mathrm{H} / \mathrm{P}$ & $+/+$ & $+/+$ & + & - & GH16 (Crh) \\
\hline 04432 & 06764 & $532 / 531$ & 92.8 & $+/-$ & $\mathrm{H} / \mathrm{H}$ & $\mathrm{P} / \mathrm{H}$ & $+/+$ & $+/+$ & + & 3 & GH13 (Alpha-amilase) \\
\hline 04693 & 04534 & $459 / 459$ & 97.6 & $-/+$ & $\mathrm{H}$ & $-/ \mathrm{W}$ & + & + & + & 8 & GH76 (DCW1) \\
\hline 05063 & 05192 & $443 / 506$ & 92.5 & - & $\mathrm{P} / \mathrm{P}$ & - & - & + & - & - & GT1 \\
\hline 01494 & 06960 & $470 / 471$ & 88.7 & $-/-$ & - & $\mathrm{H} / \mathrm{H}$ & $+/+$ & $+/+$ & + & - & GH76 (DCW1) \\
\hline 03286 & 03782 & $445 / 443$ & 79.4 & $-/+$ & $-/ P$ & $\mathrm{H}$ & - & + & + & 3 & GH72 (Gel) \\
\hline 07673 & 05717 & $323 / 323$ & 95.4 & - & - & $\mathrm{H} / \mathrm{W}$ & - & - & + & - & GH76 (DCW1) \\
\hline $00483^{*}$ & 01798 & $862 / 852$ & 95.6 & - & - & - & $-/-$ & $-/+-$ & $+-/-$ & - & $\mathrm{GH} 3$ \\
\hline 00795 & 02100 & $446 / 446$ & 97.3 & - & - & - & + & + & + & 3 & GH72 (Gel) \\
\hline 03601 & 01224 & $265 / 363$ & 25.7 & - & - & - & $+/-$ & - & - & - & GH16 (Crh1) \\
\hline 04617 & $04461^{*}$ & $234 / 237$ & 93.6 & - & - & - & + & $-/+$ & $+/-$ & 1 & CE5 (cutinase) \\
\hline 06344 & 03482 & $796 / 785$ & 96.5 & - & - & - & + & - & + & 5 & GH92 \\
\hline 06465 & 03359 & $230 / 230$ & 90.0 & - & - & - & + & - & + & - & GH23 (lysozyme) \\
\hline \multicolumn{12}{|c|}{ Aspartic proteases } \\
\hline 06207 & 03619 & $513 / 469$ & 70.4 & $-/+$ & $-/ P$ & $-/ H$ & $-/+$ & $-/+$ & + & 2 & Pepsin-like proteinase \\
\hline 08282 & 07583 & $481 / 481$ & 95.6 & $+/-$ & $\mathrm{P} / \mathrm{HP}$ & $\mathrm{P} / \mathrm{HP}$ & $+/+$ & $+/+$ & - & 2 & Pepsin-like protease \\
\hline \multicolumn{12}{|c|}{ Phospholipases } \\
\hline 01064 & 06610* & $1067 / 1004$ & 96.3 & - & - & - & + & + & - & $7 / 9$ & $\begin{array}{c}\text { Phospholipase D } \\
\text { Active site }\end{array}$ \\
\hline 03299 & 03796 & $1424 / 1398$ & 93.3 & - & - & - & + & + & + & $4 / 4$ & $\begin{array}{c}\text { phospholipase C X } \\
\text { domain }\end{array}$ \\
\hline \multicolumn{12}{|l|}{ Hydrophobins } \\
\hline 08524 & - & 419 & & + & L & $\mathrm{H}$ & + & + & + & - & Hydrophobin \\
\hline 07472 & $04235^{\star}$ & $133 / 133$ & 100 & - & - & $\mathrm{P} / \mathrm{P}$ & + & - & $+/+$ & 2829 & Hydrophobin 2 \\
\hline
\end{tabular}


\begin{tabular}{l} 
Table 1 (cont.) \\
\hline$P$ brasiliensis
\end{tabular}

\begin{tabular}{|c|c|c|c|c|c|c|c|c|c|c|c|}
\hline $\begin{array}{c}\text { P. brasiliensis } \\
(\mathrm{Pb})\end{array}$ & $\begin{array}{l}\text { P. Iutzii } \\
\text { (PI) }\end{array}$ & $\begin{array}{l}\text { Size (aa - } \\
\mathrm{Pb} / \mathrm{PI})\end{array}$ & $\begin{array}{l}\text { Identity } \\
(\%)\end{array}$ & $\begin{array}{l}\text { Big-PI } \\
\text { (Pb/PI) }\end{array}$ & $\begin{array}{l}\text { FragAnchor } \\
\text { (Pb/PI) }\end{array}$ & $\begin{array}{l}\text { PredGPI } \\
\text { (Pb/PI) }\end{array}$ & $\begin{array}{l}\text { DeGroot } \\
\text { (Pb/PI) }\end{array}$ & $\begin{array}{l}\text { GPI-SOM } \\
\text { (Pb/PI) }\end{array}$ & $\begin{array}{l}\text { SignalP } \\
\text { (Pb/PI) }\end{array}$ & EST & function \\
\hline \multicolumn{12}{|c|}{ Superoxide dismutases } \\
\hline 02842 & 02971 & $227 / 228$ & 74.4 & + & $\mathrm{P} / \mathrm{P}$ & $\mathrm{H}$ & $-/+$ & + & + & - & Superoxide dismutase \\
\hline 07418 & 04164 & $154 / 154$ & 95.4 & - & - & - & + & - & + & - & Superoxide dismutase \\
\hline \multicolumn{12}{|l|}{ Unknown } \\
\hline 05000 & 05158 & $184 / 184$ & 90.2 & + & $\mathrm{H}$ & $\mathrm{H}$ & + & + & + & 3 & CFEM superfamily \\
\hline 06374 & 02225 & $241 / 435$ & 19.1 & + & $\mathrm{H} / \mathrm{P}$ & $\mathrm{H} / \mathrm{P}$ & + & - & + & 46 & CFEM superfamily \\
\hline 03909 & 00918 & $135 / 135$ & 82.2 & + & $\mathrm{P} /-$ & $\mathrm{H}$ & + & + & + & $154 / 9$ & CFEM superfamily \\
\hline 05363 & 02225 & $289 / 281$ & 84.1 & + & $\mathrm{P} / \mathrm{P}$ & $\mathrm{H} / \mathrm{H}$ & + & + & $+/+$ & - & CFEM superfamily \\
\hline 00802 & 02093 & $728 / 723$ & 92.2 & + & - & $\mathrm{H} / \mathrm{P}$ & - & + & + & - & CFEM superfamily \\
\hline 03914 & 00912 & $189 / 189$ & 96.3 & + & $\mathrm{H}$ & $\mathrm{H} /-$ & + & + & + & $9 / 154$ & unknown; CRoW \\
\hline 04649 & 04490 & $228 / 231$ & 95.6 & $+/+$ & $\mathrm{H} / \mathrm{H}$ & $\mathrm{H} /+$ & $+/+$ & $+/+$ & + & 239 & Conserved unknown \\
\hline 06613 & 05264 & $245 / 248$ & 91.8 & $+/+$ & $\mathrm{H}$ & $\mathrm{H}$ & + & + & + & - & Conserved unknown \\
\hline 02867 & 02996 & $251 / 237$ & 47.6 & + & $\mathrm{H}$ & $\mathrm{H}$ & + & + & + & 856 & Conserved unknown \\
\hline 07354 & 04085 & $244 / 244$ & 98.3 & + & $\mathrm{H}$ & $\mathrm{H}$ & + & + & + & 4 & Conserved unknown \\
\hline 04509 & 07681 & $156 / 156$ & 84.0 & + & $\mathrm{H} / \mathrm{P}$ & $\mathrm{H}$ & + & + & + & 56 & Conserved unknown \\
\hline 02770 & $07767^{\star}$ & $328 / 328$ & 93.6 & $+/+$ & $\mathrm{H} / \mathrm{H}$ & $\mathrm{H} / \mathrm{H}$ & $+/+$ & $+/+$ & + & 6 & Conserved unknown \\
\hline 00497 & 01813 & $219 / 229$ & 81.3 & + & $\mathrm{H}$ & $\mathrm{H}$ & + & + & + & $31 / 16$ & Conserved unknown; \\
\hline 07758 & 05636 & $158 / 161$ & 75.9 & + & $\mathrm{P} / \mathrm{H}$ & $\mathrm{H}$ & + & + & + & 72 & Conserved unknown \\
\hline 03600 & 01225 & $196 / 195$ & 83.6 & $+/+$ & $\mathrm{P} / \mathrm{H}$ & $\mathrm{H} / \mathrm{P}$ & $+/+$ & $+/+$ & + & - & Conserved unknown \\
\hline 00984 & 07227 & $233 / 153$ & 90.2 & + & $\mathrm{P} / \mathrm{H}$ & $\mathrm{H}$ & + & + & + & 2 & Conserved unknown \\
\hline 06677 & 05334 & $233 / 227$ & 84.6 & + & $\mathrm{P}$ & $\mathrm{P}$ & + & + & + & 78 & Conserved unknown \\
\hline 03799 & 01033 & $202 / 208$ & 95.0 & - & $\mathrm{P}$ & $\mathrm{P}$ & + & + & + & 20 & Conserved unknown \\
\hline 02955 & 03079* & $281 / 281$ & 91.4 & $+/-$ & $\mathrm{L} /-$ & - & $+/-$ & $+/-$ & + & $0 / 5$ & Conserved unknown \\
\hline 03262 & 03757 & $282 / 309$ & 82.9 & + & $\mathrm{L} / \mathrm{P}$ & $\mathrm{H}$ & + & + & + & $3 / 4$ & Conserved unknown \\
\hline 05482 & 02344 & $224 / 232$ & 93.7 & + & $\mathrm{L} / \mathrm{H}$ & $P$ & + & + & + & $10 / 2$ & Conserved unknown \\
\hline 00773 & 02119 & $350 / 168$ & 70.2 & + & $\mathrm{H}$ & $\mathrm{H} / \mathrm{P}$ & - & + & + & $8 / 3$ & $\begin{array}{l}\text { Conserved unknown; } \\
\text { proline rich }\end{array}$ \\
\hline 02569 & 04829 & $217 / 220$ & 90.7 & + & $\mathrm{H}$ & $\mathrm{H}$ & - & + & + & 76 & Conserved unknown \\
\hline 01837 & 02807 & $182 / 172$ & 88.3 & $-/+$ & $-/ H$ & $-/ P$ & $-/+$ & + & + & 36 & Conserved unknown \\
\hline 00450 & 06528 & $224 / 229$ & 90.6 & $+/-$ & $\mathrm{L}$ & $\mathrm{H} /-$ & - & + & + & 1 & Conserved unknown \\
\hline 07141 & 00119 & $534 / 534$ & 96.0 & - & - & - & - & + & + & - & Conserved unknown \\
\hline
\end{tabular}




\begin{tabular}{|c|c|c|c|c|c|c|c|c|c|c|c|}
\hline \multicolumn{12}{|l|}{ Table 1 (cont.) } \\
\hline $\begin{array}{l}\text { P. brasiliensis } \\
\text { (Pb) }\end{array}$ & $\begin{array}{l}\text { P. lutzii } \\
\text { (PI) }\end{array}$ & $\begin{array}{l}\text { Size (aa - } \\
\mathrm{Pb} / \mathrm{PI})\end{array}$ & $\begin{array}{l}\text { Identity } \\
(\%)\end{array}$ & $\begin{array}{l}\text { Big-PI } \\
\text { (Pb/PI) }\end{array}$ & $\begin{array}{l}\text { FragAnchor } \\
(\mathrm{Pb} / \mathrm{PI})\end{array}$ & $\begin{array}{l}\text { PredGPI } \\
\text { (Pb/PI) }\end{array}$ & $\begin{array}{l}\text { DeGroot } \\
\text { (Pb/PI) }\end{array}$ & $\begin{array}{l}\text { GPI-SOM } \\
\text { (Pb/PI) }\end{array}$ & $\begin{array}{c}\text { SignalP } \\
(\mathrm{Pb} / \mathrm{PI})\end{array}$ & EST & function \\
\hline 02604 & 04863 & $367 / 389$ & 87.1 & - & $\mathrm{H} / \mathrm{P}$ & $\mathrm{H}$ & + & + & + & $1 / 1$ & Conserved unknown \\
\hline 01394 & 06493 & $273 / 272$ & 89.3 & - & $\mathrm{H}$ & $P$ & - & + & + & 10 & Conserved unknown \\
\hline 02487 & 04745 & $441 / 436$ & 89.9 & - & $\mathrm{H}$ & $P$ & - & + & + & - & Conserved unknown \\
\hline 02535 & 04795 & $121 / 121$ & 86.8 & - & $\mathrm{L}$ & - & + & + & - & $32 / 43$ & Conserved unknown \\
\hline 06557 & 09093 & $727 / 724$ & 85.6 & - & $-/ P$ & $\mathrm{H}$ & + & + & + & - & Conserved unknown \\
\hline 06704 & 05361 & $600 / 628$ & 95.0 & - & - & $\mathrm{P} /(\mathrm{W})$ & + & + & + & - & Conserved unknown \\
\hline 04289 & 08312 & $204 / 204$ & 97.0 & $-1+$ & - & $\mathrm{P}$ & + & + & + & $86 / 85$ & Conserved unknown \\
\hline 04623 & 03197 & $308 / 492$ & 37.3 & - & - & W/- & - & - & + & - & Conserved unknown \\
\hline 07877 & 00250 & $126 / 126$ & 100.0 & - & - & - & + & - & + & - & Conserved unknown \\
\hline 00455 & 06522 & $509 / 508$ & 95.6 & - & - & - & + & $\mathrm{U} /+$ & + & - & $\begin{array}{c}\text { Conserved unknown; } \\
\text { DUF185 }\end{array}$ \\
\hline $06524^{*}$ & 08951 & $437 / 437$ & 89.2 & - & - & - & $-/-$ & $-/-$ & $-/-$ & - & Conserved unknown \\
\hline 04816 & 00701 & $149 / 322$ & 23.4 & - & - & - & $+/-$ & & + & - & $\begin{array}{c}\text { Conserved unknown; } \\
\text { DUF3435 }\end{array}$ \\
\hline 03732 & 01098 & $423 / 337$ & 89.0 & - & - & - & + & + & + & $9 / 8$ & Conserved unknown \\
\hline 00118 & 04530 & $162 / 329$ & 16.0 & - & - & - & $+/-$ & $\mathrm{U} /-$ & + & - & Conserved unknown \\
\hline 02815 & 07721 & $128 / 190$ & 23.4 & - & - & - & $+/-$ & - & + & - & Conserved unknown \\
\hline 00406 & 01364 & $125 / 127$ & 65.6 & - & - & - & $-/-$ & $-/-$ & $-/-$ & - & Conserved unknown \\
\hline 08385 & 08073 & $139 / 139$ & 96.4 & + & $\mathrm{H}$ & $\mathrm{H}$ & + & + & - & 72 & Conserved unknown \\
\hline 03926 & 00898 & $178 / 178$ & 93.2 & + & $P$ & W & - & + & - & - & Conserved unknown \\
\hline 03750 & $01075^{\star}$ & $517 / 505$ & 96.2 & $+/ P$ & $\mathrm{P} / \mathrm{P}$ & - & $+/+$ & - & - & 3 & Conserved unknown \\
\hline $06896^{*}$ & 02575 & $398 / 450$ & 94.4 & $+/+$ & - & - & $+/+$ & $+/+$ & - & - & $\begin{array}{c}\text { Conserved unknown; } \\
\text { DUF3722 }\end{array}$ \\
\hline 07778 & $05614^{*}$ & $396 / 289$ & 95.1 & $+/+$ & - & $-/-$ & $+/+$ & +/+ & - & $11 / 13$ & Conserved unknown \\
\hline 00058 & 01719 & $98 / 435$ & 68.4 & $+/-$ & $+/-$ & $+/-$ & $+/-$ & $+/-$ & - & 1 & Conserved unknown \\
\hline $05186^{*}$ & 07493 & $518 / 429$ & 90.6 & + & - & - & - & + & - & - & Conserved unknown \\
\hline 03942 & 00890 & $304 / 304$ & 96.0 & - & $\mathrm{P}$ & $\mathrm{P}$ & & + & - & 1 & $\begin{array}{c}\text { Conserved unknown; } \\
\text { DUF1640 }\end{array}$ \\
\hline 03510 & 01314 & $97 / 97$ & 96.9 & - & $\mathrm{P}$ & - & + & + & - & - & Conserved unknown \\
\hline 01219 & 06319* & $723 / 697$ & 91.8 & - & $\mathrm{L} /(\mathrm{L})$ & - & $+/+$ & $+/+$ & - & - & Conserved unknown \\
\hline 03777 & 01053 & $686 / 686$ & 95.3 & - & $\mathrm{H}$ & - & - & + & - & - & Conserved unknown \\
\hline
\end{tabular}




\begin{tabular}{|c|c|c|c|c|c|c|c|c|c|c|c|}
\hline 00231 & 01547 & $263 / 263$ & 97.3 & - & $\mathrm{H}$ & - & - & - & - & - & Conserved unknown \\
\hline $06057^{*}$ & 05937 & $424 / 421$ & 94.0 & - & $-/-$ & - & - & $-/-$ & - & - & Conserved unknown \\
\hline 04535 & 04363 & $142 / 370$ & 40.8 & - & L/- & - & - & $+/-$ & - & - & Conserved unknown \\
\hline 00551 & 01876 & $756 / 736$ & 90.4 & - & L/- & - & - & $+/ U$ & - & - & Conserved unknown \\
\hline 05519 & - & 67 & & - & $\mathrm{L}$ & - & + & - & - & - & Conserved unknown \\
\hline 08345 & 08031 & $303 / 266$ & 86.8 & - & L/- & - & - & $+/-$ & - & - & Conserved unknown \\
\hline 02992 & $03117^{*}$ & $451 / 484$ & 90.0 & $-/+$ & - & $\mathrm{H} /-$ & + & + & - & - & Conserved unknown \\
\hline 01591 & 07061 & $591 / 568$ & 92.9 & - & - & - & + & $-/+$ & - & - & $\begin{array}{c}\text { Conserved unknown; } \\
\text { DUF3480 }\end{array}$ \\
\hline 04859 & $05011^{*}$ & $142 / 142$ & 87.3 & - & - & - & + & - & - & - & Conserved unknown \\
\hline 05013 & 05173 & $214 / 210$ & 93.3 & - & - & - & $+/-$ & $+/-$ & - & - & Conserved unknown \\
\hline 05708 & 05555 & $974 / 1226$ & 88.9 & - & - & - & + & - & - & - & Conserved unknown; \\
\hline 07277 & 08671 & $488 / 503$ & 40.1 & - & - & - & $+/-$ & $+/-$ & - & - & Conserved unknown \\
\hline 04284 & 07517 & $59 / 130$ & 22.0 & - & - & - & + & - & - & - & Conserved unknown \\
\hline 07635 & 05753 & $565 / 565$ & 94.3 & - & - & $\mathrm{P} / \mathrm{W}$ & $-/-$ & + & - & - & Conserved unknown \\
\hline 07754 & - & 78 & & - & - & - & + & - & - & - & Conserved unknown \\
\hline $08596^{*}$ & 09050 & $336 / 333$ & 87.3 & - & - & - & $-/-$ & + & - & - & Conserved unknown \\
\hline
\end{tabular}

$\mathrm{HP}=$ Highly Probable; $\mathrm{P}=$ Probable $; \mathrm{L}=$ Low Probable; $\mathrm{W}=$ Weakly Probable SignallP: + secretion signal, - no secretion signal not present

$\mathrm{GH}$ : glycosylhydrolase 


\begin{tabular}{|c|c|c|c|c|c|c|c|c|c|c|c|c|c|c|c|c|c|c|c|}
\hline $\mathbb{2}$ & $\bar{a}$ & 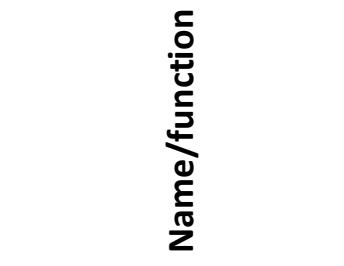 & 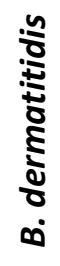 & 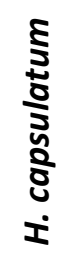 & 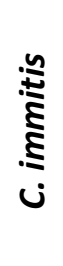 & $\begin{array}{l}\stackrel{n}{\mathbf{s}} \\
\mathbf{S} \\
\dot{\Sigma}\end{array}$ & 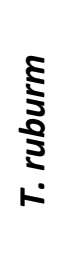 & $\begin{array}{l}\frac{n}{\delta} \\
\frac{\delta}{5} \\
\frac{0}{5} \\
\dot{\leftarrow}\end{array}$ & 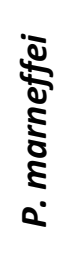 & 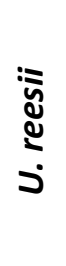 & 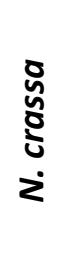 & 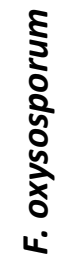 & 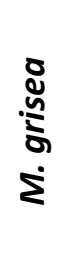 & 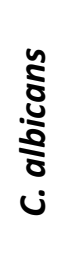 & 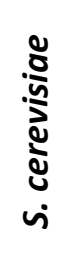 & 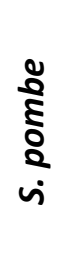 & 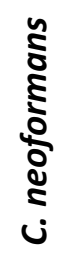 & 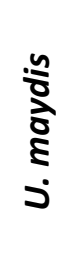 & 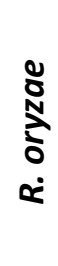 \\
\hline \multicolumn{20}{|l|}{ ORF } \\
\hline PADG_07418 & PAAG_04164 & SOD & & & & & & & & & & & & & & & & & \\
\hline PADG_06688 & PAAG_05346 & peptidase & & & & & & & & & & & & & & & & & \\
\hline PADG_00400 & PAAG_01372 & GH3 & & & & & & & & & & & & & & & & & \\
\hline PADG_00483 & PAAG_01798 & $\mathrm{GH} 3$ & & & & & & & & & & & & & & & & & \\
\hline PADG_02814 & PAAG_07722 & PLC & & & & & & & & & & & & & & & & & \\
\hline PADG_03306 & PAAG_03802 & GT62 & & & & & & & & & & & & & & & & & \\
\hline PADG_02190 & PAAG_00495 & GT2 & & & & & & & & & & & & & & & & & \\
\hline PADG_05076 & PAAG_05245 & GH72 & & & & & & & & & & & & & & & & & \\
\hline PADG_05063 & PAAG_05192 & GT1 & & & & & & & & & & & & & & & & & \\
\hline PADG_04299 & PAAG_06899 & $\mathrm{GH} 47$ & & & & & & & & & & & & & & & & & \\
\hline PADG_01954 & PAAG_02926 & SOD & & & & & & & & & & & & & & & & & \\
\hline PADG_06700 & PAAG_05357 & $\mathrm{GH} 28$ & & & & & & & & & & & & & & & & & \\
\hline PADG_06728 & PAAG_05380 & $\mathrm{GH} 20$ & & & & & & & & & & & & & & & & & \\
\hline PADG_03299 & PAAG_03796 & PLC & & & & & & & & & & & & & & & & & \\
\hline PADG_03286 & PAAG_03782 & GH72 & & & & & & & & & & & & & & & & & \\
\hline PADG_04918 & PAAG_05068 & $\mathrm{GH} 2$ & & & & & & & & & & & & & & & & & \\
\hline PADG_06207 & PAAG_03619 & Aspartyl protease & & & & & & & & & & & & & & & & & \\
\hline PADG_04693 & PAAG_04534 & GH76 & & & & & & & & & & & & & & & & & \\
\hline PADG_04499 & PAAG_07670 & ECM33 & & & & & & & & & & & & & & & & & \\
\hline PADG_04416 & PAAG_06779 & GH16 & & & & & & & & & & & & & & & & & \\
\hline PADG_04328 & PAAG_06867 & GH16 & & & & & & & & & & & & & & & & & \\
\hline PADG_04617 & PAAG_04461 & CE5 & & & & & & & & & & & & & & & & & \\
\hline PADG_04435 & - & cutinase & & & & & & & & & & & & & & & & & \\
\hline PADG_04432 & PAAG_06764 & $\alpha$-amilase & & & & & & & & & & & & & & & & & \\
\hline PADG_00795 & PAAG_02100 & GH72 & & & & & & & & & & & & & & & & & \\
\hline PADG_06344 & PAAG_03482 & GH92 & & & & & & & & & & & & & & & & & \\
\hline PADG_07472 & PAAG_04235 & Hydrophobin2 & & & & & & & & & & & & & & & & & \\
\hline PADG_01494 & PAAG_06960 & GH76 & & & & & & & & & & & & & & & & & \\
\hline PADG_02842 & PAAG_02971 & SOD & & & & & & & & & & & & & & & & & \\
\hline PADG_08282 & PAAG_07583 & Aspartyl protease & & & & & & & & & & & & & & & & & \\
\hline PADG_07673 & PAAG_05717 & GH76 & & & & & & & & & & & & & & & & & \\
\hline PADG_00012 & PAAG_01763 & Carboxypeptidase & & & & & & & & & & & & & & & & & \\
\hline PADG_04195 & PAAG_00636 & Dioxygenase & & & & & & & & & & & & & & & & & \\
\hline PADG_07620 & PAAG_05768 & Unknown? & & & & & & & & & & & & & & & & & \\
\hline PADG_01014 & PAAG_06556 & Poly (ADP-ribose) glycohydrolase & & & & & & & & & & & & & & & & & \\
\hline PADG_08524 & - & hydrophobin & & & & & & & & & & & & & & & & & \\
\hline PADG_02460 & PAAG_04708 & Pga1 & & & & & & & & & & & & & & & & & \\
\hline & & & & & & & & & & & & & & & & & & & \\
\hline
\end{tabular}

Fig 1. Presence of homologs for GPI proteins with known function.

GT62 = $\alpha-1-6$ mannosyltransferase

GT2 = dolichyl-phosphate beta-glucosyltransferase 
bioRxiv preprint doi: https://doi.org/10.1101/347351; this version posted June 15,2018 . The copyright holder for this preprint (which was not certified by peer review) is the author/funder. All rights reserved. No reuse allowed without permission.
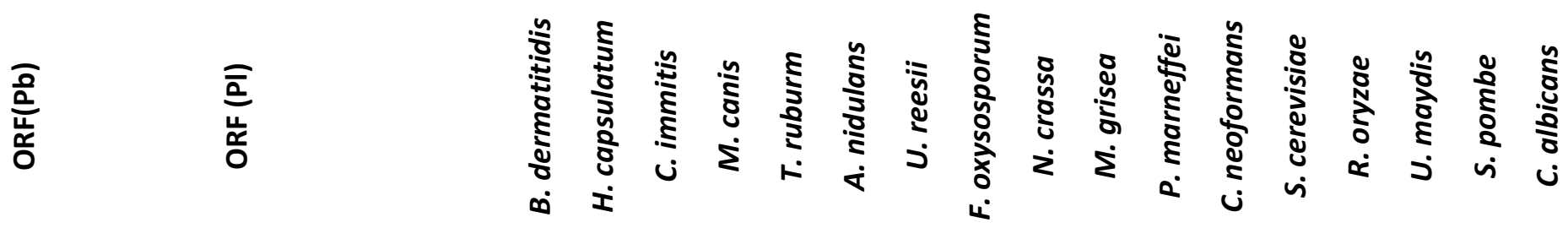

\begin{tabular}{|c|c|}
\hline PADG_03942 & PAAG_00890 \\
\hline PADG_03417 & PAAG_03924 \\
\hline PADG_03750 & PAAG_01075 \\
\hline PADG_07354 & PAAG_04085 \\
\hline PADG_04289 & PAAG_08312 \\
\hline PADG_04649 & PAAG_04490 \\
\hline PADG_05000 & PAAG_05158 \\
\hline PADG_01394 & PAAG_06493 \\
\hline PADG_03691 & PAAG_01139 \\
\hline PADG_03732 & PAAG_01098 \\
\hline PADG_01219 & PAAG_06319 \\
\hline PADG_05482 & PAAG_02344 \\
\hline PADG_06704 & PAAG_05361 \\
\hline PADG_06613 & PAAG_05264 \\
\hline PADG_07778 & PAAG_05614 \\
\hline PADG_02535 & PAAG_04795 \\
\hline PADG_03015 & PAAG_03134 \\
\hline- & PAAG_07679 \\
\hline PADG_00119 & PAAG_01656 \\
\hline PADG_07758 & PAAG_05636 \\
\hline PADG_6374 & - \\
\hline PADG_02955 & PAAG_03079 \\
\hline PADG_04717 & PAAG_04558 \\
\hline PADG_03277 & PAAG_03775 \\
\hline PADG_02569 & PAAG_04829 \\
\hline PADG_04509 & PAAG_07681 \\
\hline PADG_02867 & PAAG_02996 \\
\hline PADG_08385 & PAAG_08073 \\
\hline PADG_02604 & PAAG_04863 \\
\hline PADG_03909 & PAAG_00918 \\
\hline PADG_01837 & PAAG_02807 \\
\hline PADG_00497 & PAAG_01813 \\
\hline PADG_02487 & PAAG_04745 \\
\hline PADG_03799 & PAAG_01033 \\
\hline PADG_06677 & PAAG_05334 \\
\hline PADG_06418 & PAAG_03418 \\
\hline PADG_05363 & PAAG_02225 \\
\hline PADG_02992 & PAAG_03117 \\
\hline PADG_00802 & PAAG_02093 \\
\hline PADG_00984 & PAAG_07227 \\
\hline PADG_03926 & PAAG_00898 \\
\hline PADG_00450 & PAAG_06528 \\
\hline PADG_00776 & PAAG_02117 \\
\hline PADG_03914 & PAAG_00912 \\
\hline PADG_06557 & PAAG_09093 \\
\hline PADG_05279 & PAAG_08515 \\
\hline PADG_02770 & PAAG_07767 \\
\hline PADG_03262 & PAAG_03757 \\
\hline PADG_03673 & PAAG_01156 \\
\hline PADG_03600 & PAAG_01225 \\
\hline PADG_00058 & PAAG_01719 \\
\hline PADG_00773 & PAAG_02119 \\
\hline
\end{tabular}

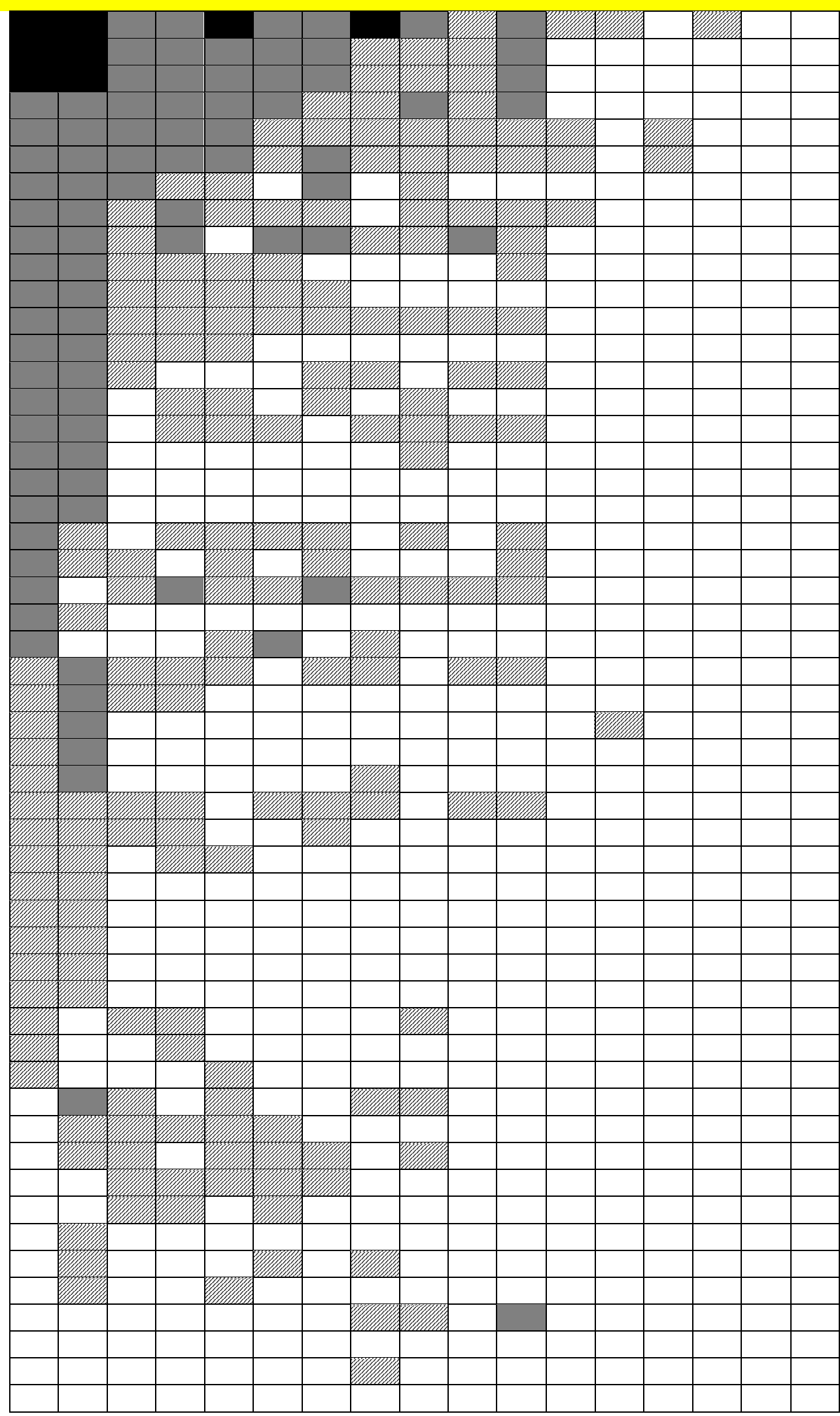

Fig 1. Presence of homologs for GPI proteins with unknown function. 